\title{
Photoluminescence Properties of Novel Fluorescent Polyimide Based on Excited State Intramolecular Proton Transfer at The End Groups
}

\author{
Naiqiang Liang, Eisuke Fujiwara, Mayuko Nara, Ryohei Ishige, and Shinji Ando* \\ Department of Chemical Science and Engineering, Tokyo Institute of Technology, \\ O okayama 2-12-1-E4-5, M eguro-ku, Tokyo 152-8552, Japan \\ *sando@ polymer.titech.ac.jp
}

\begin{abstract}
Excited state intramolecular proton transfer (ESIPT) is one of the distinctive photophysical processes of fluorescent compounds and polymers, which induces enhanced fluorescence with a very large Stokes shift. In this work, 6FDA/DCHM/3HPA polyimides (PIs) having bulky $-\mathrm{CF}_{3}$ groups were synthesized, in which $3 \mathrm{HPA}$ is an end-group which exhibits ESIPT with enhanced green emission when irradiated by UV light at $365 \mathrm{~nm}$. Introduction of bulky side chains can improve fluorescent properties via suppression of aggregation induced quenching as well as intermolecular interactions. All the 6FDA-based PI films were colorless and transparent in the visible region, and the PIs having 3HPA end-groups exhibited obvious green emission at $530 \mathrm{~nm}$, when excited at $340 \mathrm{~nm}$. Compared with the corresponding ODPA-based PIs which have no bulky side groups, the 6FDA-based PIs demonstrated significantly higher quantum yields in the solid state $(\Phi=$ $0.14-0.25$ ), which is attributable to the looser aggregation structures owing to the bulky and less polarizable $-\mathrm{CF}_{3}$ group and the absence of energy transfer to/from the non-fluorescent main chain units. Accordingly, the experimental results proved that the introduction of bulky $-\mathrm{CF}_{3}$ groups using $6 \mathrm{FDA}$ moiety is a promising way to create highly fluorescent end-capped PIs.
\end{abstract}

Keywords: Polyimide, Fluorescence, ESIPT, Stokes shift, Energy transfer

\section{Introduction}

Polyimides (PIs) are a group of condensation polymers which have high thermal stability, good mechanical strength, excellent chemical stability, low dielectric constant, and low thermal expansion [1-8]. Thus, PIs have been widely used as insulating interlayer films in microelectronic industry and thermal controlling films in aerospace industry. Recently, PIs have attracted much more attention because of their excellent properties such as 1) good film-forming properties, 2) excellent comprehensive performance; outstanding thermal stability and environment stability, 3) structural diversity based on plenty of commercialized dianhydrides and diamine monomers [3].

Since PIs generally exhibit poor solubility in organic solvents, a conventional two-step synthetic method is adopted to form

Received April 1,2019

Accepted April 15, 2019 five-membered imide rings: 1) dianhydride and diamine reaction in aprotic polar solvents at ambient temperature to form polyamic acid (PAA), 2) ring closure of PAA (i.e. imidization) by heating or chemical dehydration after film formation from the PAA solution [3]. Because of the poor solubility of PIs, optical characteristics of the PIs, such as absorption and fluorescence properties, can be evaluated only in the solid state. However, the formation of aggregation, charge-transfer (CT) complex, and oriented structures in solid samples make interpretation of the optical properties difficult [4].

In order to clarify the fluorescent properties of PIs, imide model compounds, whose structures are almost same as the repeating units of the PIs, have been synthesized, and the fluorescent properties of PIs were investigated through the analysis of the model compounds [8]. Demeter et 
al. [9] have reported the fluorescent properties of some N-phenylnaphthalimide derivatives, which suggested that the structural relaxation of the Frank-Condon state and rearrangement of solvent molecules yield locally excited (LE) and charge transfer (CT) states. The LE state shows a shorter wavelength emission compared to the $\mathrm{CT}$ state. However, owing to the low oscillator strength originated from the spatially separated orbitals of HOMO and LUMO, florescent emission from the CT state always results in low photoluminescent efficiency [10]. Furthermore, aromatic PIs with strong CT transitions exhibit strong coloration, which is undesirable for practical applications of the florescent PIs [4].

We have reported that the use of alicyclic diamines can effectively suppress the CT transitions in PIs with keeping the LE transitions, which can significantly improve the photoluminescent efficiency [8]. For example, fully aromatic PMDA/ODA PI shows only very weak florescence from the excited CT state with a low quantum yield of $10^{-6}$ [10]. However, SBPDA/t-CHDA PI containing a cyclohexane ring in the main chain showed obvious blue florescent from the LE state with a higher quantum yield of about 0.05 [11].

Even though semi-aliphatic PIs have good fluorescent properties, most of semi-aliphatic PIs with LE emission can emit only blue florescence with a small Stokes shift, which corresponds to a small difference between the excitation and emission wavelengths. For example, the Stokes shift of a semi-aliphatic HQDEA/DCHM PI was $5199 \mathrm{~cm}^{-1}$ [8]. For practical applications of fluorescent PIs such as solar spectral converters which convert UV light to longer wavelength visible light [12], Stokes shifts larger than 8000 $\mathrm{cm}^{-1}$ are demanded. We have reported that introduction of fluorine atoms into the skeletal structures of dianhydrides can provide green (10FEDA/DCHM PI), and even red (P2FEDA/DCHM PI) fluorescent PIs [13]. This is because the electron affinity of these dianhydrides are significantly enhanced by the strong electron-withdrawing property of fluorine atoms. However, these films exhibit strong absorption bands in the visible region, which causes obvious and deep coloration. In other words, the Stokes shifts of these conventional PIs are still not large enough.

There are several strategies to obtain fluorescent PIs exhibiting large Stokes shifts caused by structural relaxation in the excited state. Synthesis and use of novel dianhydrides having a function of excited state intramolecular proton transfer (ESIPT) is an effective way to develop highly fluorescent PI with a large Stokes shift [14]. In general, ESIPT moiety requires an intramolecular hydrogen bond (HB) between a proton donor and a proton acceptor, which are located within a close distance in a molecule to form HB [15]. In the ground state, ESIPT molecule exists as an enol form (E) which is stabilized by the intramolecular HB. After excitation by UV irradiation, the acidity of the proton donor and the basicity of the proton acceptor are increased in the excited state, and then a hydrogen atom transfers from the donor to the acceptor via intramolecular HB. The excited molecule is immediately transformed to a keto form $(\mathrm{K})$. Followed by radiative deactivation to the ground state, reverse proton transfer occurs, and the molecule goes back to the initial enol form. In general, difference in species between the as-excited state $\left(E^{*}\right)$ and the structurally relaxed emissive state $\left(\mathrm{K}^{*}\right)$ gives rise to a large Stokes shift. ESIPT process has attracted much attention due to the following reasons: 1) emission of large Stokes-shifted fluorescence (6000-12000 $\mathrm{cm}^{-1}$ ) without self-absorption, 2) efficient photocycle process $\left(E-E^{*}-K^{*}-K-E\right)$ without side reactions, 3) extremely fast process (reaction rate: $k(E S I P T)>10^{12} \mathrm{~S}^{-1}$ ) which means that ESIPT occur even at very low temperatures and has priority when competing with other photophysical processes [15].

Previously, our group has successfully prepared a series of PIs end-capped with ESIPT moieties (ODPA/DCHM/3HPA PIs), which showed prominent green fluorescence [1]. The homo-PI of ODPA/DCHM and the end group of 3HPA inherently show blue and green fluorescence at 410 and $530 \mathrm{~nm}$, respectively. In contrast, the ODPA/DCHM/3HPA PIs exhibited green fluorescence peaks only at any contents of the end-groups, which is attributable to ESIPT of the end-groups directly excited or excited through the efficient energy transfer from the main chain. In this case, the end-capped PIs showed large Stokes-shifted emission as well as good optical transparency, which provides us a good strategy to prepare highly transparent and fluorescent PIs.

On the other hand, He et al. [16] have reported that introduction of bulky groups can enhance the fluorescent properties because of the reduced 


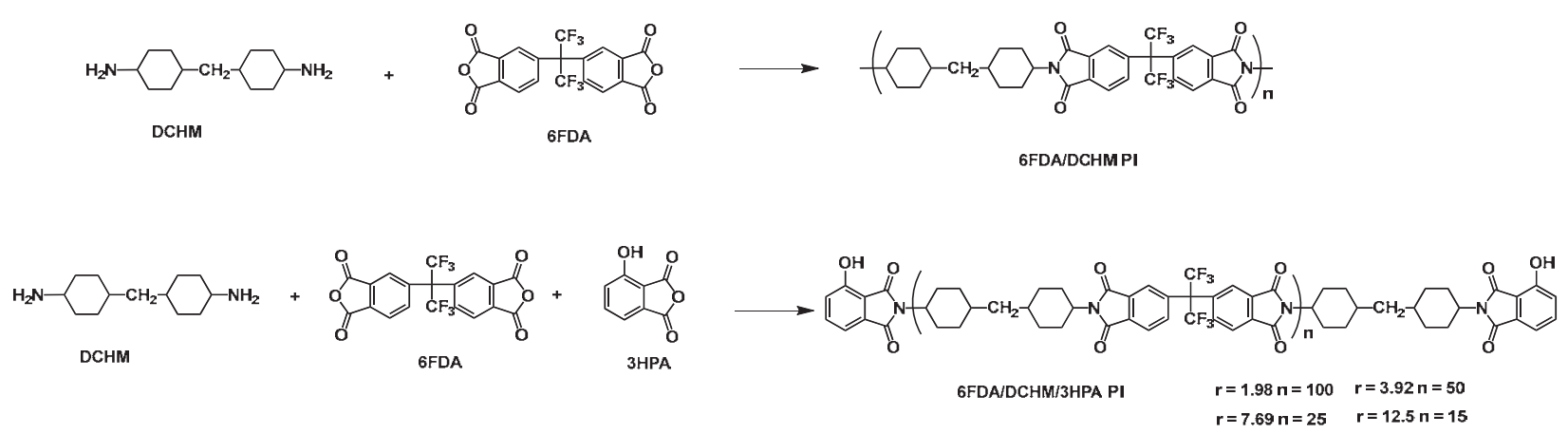

Fig. 1. Synthetic schemes of non-fluorescent 6FDA/DCHM and highly fluorescent 6FDA/DCHM/3HPA PIs.

interchain interactions and self-quenching. As mentioned above, since one of the advantages of fluorescent PIs is its structure diversity, there are plenty of commercialized monomers can be chosen to prepare the PIs. Thus, in this study, in order to improve the photoluminescent properties of PIs having 3HPA end-groups, 6FDA dianhydride has been used because of its bulky and less polarizable trifluoromethyl $\left(-\mathrm{CF}_{3}\right)$ groups. In addition, the strong electron withdrawing property of fluorine atoms is effectively blocked by the quaternary carbon, $\mathrm{C}\left(\mathrm{CF}_{3}\right)_{2}-$. Moreover, 6FDA endows PIs with good optical transparency, low dielectric constant, and low refractive indices [17]. Thus, in this report, 6FDA/DCHM/3HPA PIs (Fig. 1) have been synthesized, and their fluorescent properties were precisely measured and investigated.

\section{Experimental}

\subsection{Materials}

Hexafluoroisopropylidene-2,2-bisphthalic-anh ydride (6FDA) purchased from TCI (Tokyo Chemical Industry, Co. Ltd.) was used after sublimation under reduced pressure. 3-Hydroxyphthalicanhydride (3HPA) purchased from Aldrich was used as received. 4,4'-Diaminocyclohexylmethane (DCHM) purchased from TCI was used after recrystallization from $n$-hexane followed by sublimation under reduced pressure. $\mathrm{N}, 0$-Bis(trimethylsilyl)trifluoro- acetamide (99+\%, BSTFA) and N,N-dimethylacetamide (anhydrous, DMAc) were purchased from Aldrich and used as received.

\subsection{Synthesis and preparation of PI films}

The precursors of 6FDA/DCHM PIs, poly(amic acid) silylesters (PASEs), were prepared by in situ silylation method as reported [18-20]. In case of 6FDA/DCHM PI, firstly,
DCHM and 1.05 molar equivalent of BSTFA were dissolved in DMAc and stirred for $30 \mathrm{~min}$. Then, an equimolar 6FDA dianhydride to DCHM was added and stirred for $48 \mathrm{~h}$ to give a PASE solution. Trimethylsilylation of amino groups of the diamines by BSTFA can avoid salt formation between unreacted amino and carboxyl groups which results in insoluble precipitation.

For the end-capped PIs of 6FDA/DCHM/3HPA, the composition of PIs was determined by the following equations (1) and (2). The equations (1) and (2) are considered to hold only if the amount of 6FDA is equal to that of DCHM and the reactivity of 6FDA and DCHM was 100\%.

$$
\begin{aligned}
& r=\frac{100 \times m(\text { end })}{m(\text { end })+2 m(6 F D A)} \\
& n=-1+\frac{200}{r}
\end{aligned}
$$

where $r, n, m(e n d), m(6 F D A)$ represent the molar fraction of reactive groups in the end groups (3HPA), the number average degree of polymerization of PASE, the molar number of reactive groups in 3HPA end group, and those of 6FDA dianhydride, respectively. In this paper, $r$ values were set to $1.98,3.92,7.69$, and 12.5 , which correspond to $\mathrm{n}=100,50,25$, and 15 , respectively. The amounts of diamine, dianhydride and end group were calculated based on Eq. (1). The preparation procedure of trimethylsilylated DCHM solution was same as that of 6FDA/DCHM PASE. After addition of 6FDA to the DCHM solution and stirred at room temperature for $24 \mathrm{~h}, 3 \mathrm{HPA}$ anhydride was added and further stirred for $48 \mathrm{~h}$ to afford PASE solution. Reaction of the anhydride with amino-termini of PASE main chain gave a terminal-modified PASE main chain.

PI films were formed by spin coating of PASE solution on fused silica (amorphous $\mathrm{SiO}_{2}$ ) 
substrates, followed by drying at $70{ }^{\circ} \mathrm{C}$ for $1 \mathrm{~h}$ and subsequent thermal imidaization at $220^{\circ} \mathrm{C}$ for 1.5 $h$. All the heating procedure was conducted under nitrogen flow, and the temperature increasing rate was $3.0^{\circ} \mathrm{C} / \mathrm{min}$.

\subsection{Measurements}

UV/vis absorption spectra of PI films formed on fused-silica substrates were measured by JASCO V-670 spectrometer (JASCO Corp., Japan). The UV/vis absorption spectra were normalized by film thickness. Excitation/emission spectra of PI films were measured by a Hitachi F-7100 fluorescence spectrometer (Hitachi high-technology Corp., Japan): the emission spectra were measured with excitation at the peak wavelengths $\left(\lambda_{\text {ex }}\right)$ of corresponding excitation spectra. In contrast, the excitation spectra were measured by monitoring the fluorescence intensities at the peak wavelengths $(\lambda \mathrm{em})$ of emission spectra. Photoluminescence quantum yield $(\Phi)$ of the PI films was measured by using a calibrated integrating sphere (HAMAMATSU C9920) connected to a multi-channel analyzer (HAMAMATSU C7473) via an optical fiber link. In this measurement, PI films were excited at a controlled $\lambda_{\text {ex }}$ by a monochromated Xenon light source. Fluorescence lifetimes of PI films were acquired with a HAMAMATSU fluorescence lifetime instruments (Quantaurus-Tau, C11367-24).

\section{Results and discussion}

6FDA dianhydride have been widely used for

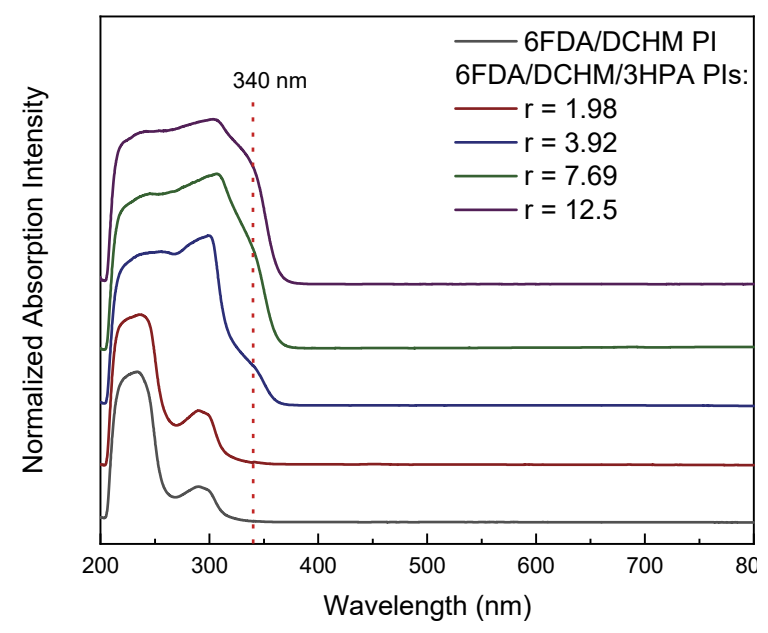

Fig. 2. UV-vis absorption spectra of 6FDA/DCHM PI and 6FDA/DCHM/3HPA PIs, each spectrum is shifted vertically for visibility.

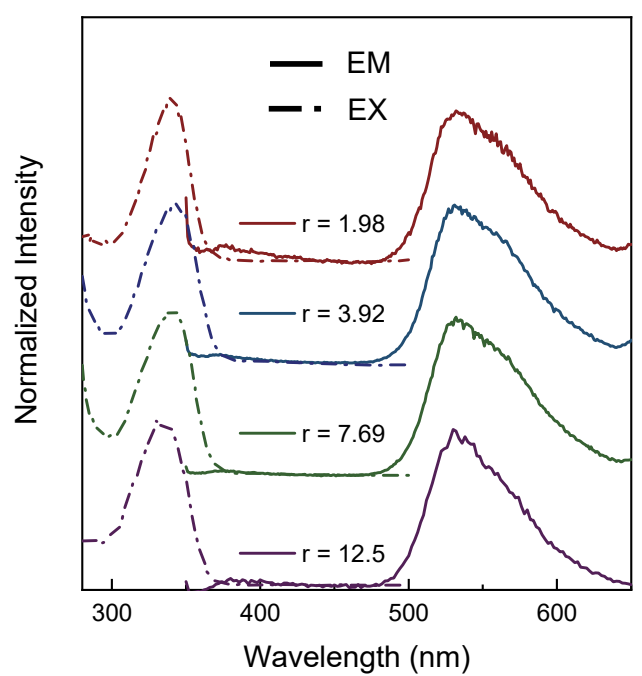

Fig. 3. Excitation/emission spectra of end-capped 6FDA/ DCHM/3HPA PIs.

PI synthesis because it endows the PIs with low dielectric constant, good solubility in polar solvents, and high gas permeability. In particular, 6FDA-based PIs exhibit high optical transparency because of its low electron affinity $\left(E_{A}\right)$ which reduces intra- and interchain charge transfer (CT) transition [6]. Figure 2 displays the UV/Vis absorption spectra of 6FDA-based PI films, in which the absorbance was normalized by the film thickness. The 6FDA/DCHM PI shows two absorption peaks in the UV region at 240 and 280 $\mathrm{nm}$, whereas no other absorption peaks were observed above $320 \mathrm{~nm}$. The excellent transparency in the near-UV and visible regions indicates that not only the intra-molecular CT but also inter-molecular $\mathrm{CT}$ interactions are effectively suppressed by looser molecular packing of 6FDA-based PIs. These absorption peaks are also observed for the end-capped 6FDA/DCHM/3HPA PIs. It should be noted that a new absorption peak was founded at $340 \mathrm{~nm}$ in the 6FDA/DCHM/3HPA PIs, which is attributable to the absorption of 3HPA end groups [12]. Hence, it is reasonable that the peak

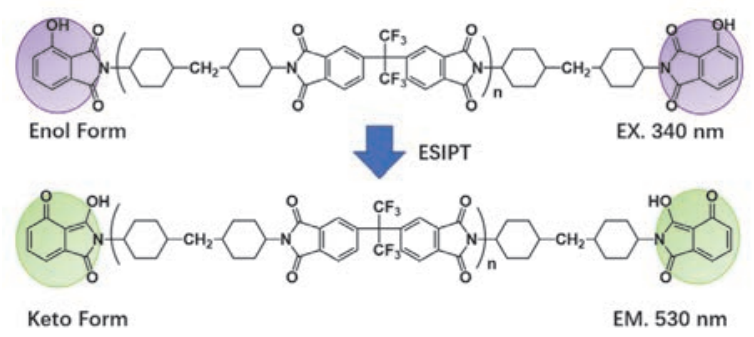

Fig. 4. Schematic representation of the excitation and emission mechanism with ESIPT process in 6FDA/DCHM/3HPA PI. 


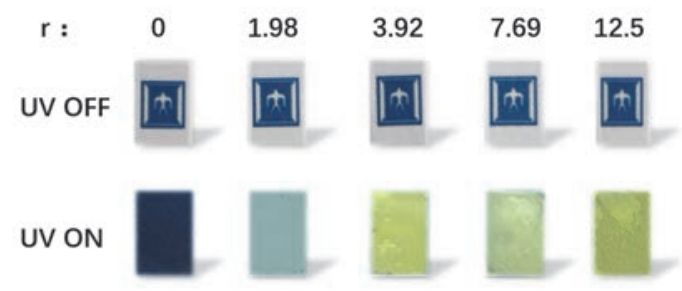

Fig. 5. Photo images of $6 \mathrm{FDA} / \mathrm{DCHM}$ PI and 6FDA/DCHM/3HPA PIs with and without UV light irradiation.

intensity at $340 \mathrm{~nm}$ (indicated by the dashed line in Fig. 2) gradually increased against the 3HPA end group ratio $(r)$.

Figure 3 demonstrates the excitation (dashed line) and emission spectra (solid line) of 6FDA/DCHM/3HPA PIs. An enhanced green fluorescence emission was observed at $530 \mathrm{~nm}$ when excited at $340 \mathrm{~nm}$, which displays a significantly large Stokes shift $\left(10,544 \mathrm{~cm}^{-1}\right)$. Since the electron transition of 6FDA/DCHM PI by UV light is assigned to non-fluorescent $n-\pi^{*}$ transition [6], the obvious green fluorescence

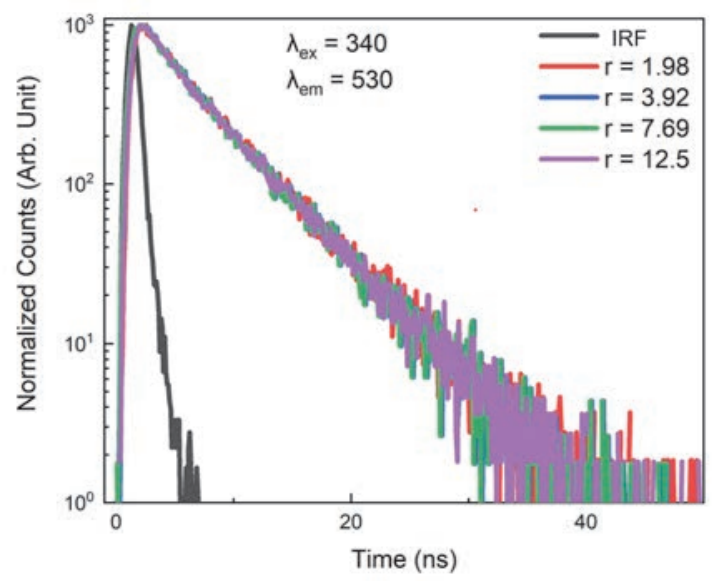

Fig. 6. Fluorescence decay curves at $530 \mathrm{~nm}$ of 6FDA/DCHM/3HPA PIs measured with excitation at $340 \mathrm{~nm}$. Instrument response function (IRF) is shown as black line.

Table 1. Fluorescence life time $(\tau)$ of 6FDA/DCHM/3HPA PI with different end group fractions ( $r$ ) and degrees of polymerization (n).

\begin{tabular}{ccc}
\hline$r$ & $\mathrm{n}$ & $\tau(\mathrm{ns})$ \\
\hline 1.98 & 100 & 4.99 \\
3.92 & 50 & 4.90 \\
7.69 & 25 & 4.94 \\
12.5 & 15 & 4.90 \\
\hline
\end{tabular}

Table 2. End group fraction $r$, the degree of polymerization $\mathrm{n}$, and fluorescent quantum yield $\Phi$ of the 6FDA- and ODPA-based PIs. (excited at 340 nm) [1].

\begin{tabular}{ccc}
\hline PI & $r$ & $\Phi$ \\
\hline & 1.98 & 0.14 \\
6FDA/DCHM/3HPA & 3.92 & 0.25 \\
& 7.69 & 0.22 \\
& 12.5 & 0.21 \\
\hline & 1.98 & 0.09 \\
ODPA/DCHM/3HPA & 3.92 & 0.11 \\
& 7.69 & 0.13 \\
& 12.5 & 0.14 \\
\hline
\end{tabular}

should be emitted from the 3HPA end group. The excitation and emission peaks of the 6FDA/DCHM/3HPA PIs with different $r$ ratios are similar to those of 3HPA model compound as reported previously [1]. Therefore, the large Stokes-shifted fluorescence of the 6FDA/DCHM/3HPA PIs derived from the ESIPT process (ESIPT fluorescence) which takes place at the 3HPA end groups as shown in Fig. 4.

The photo images of the 6FDA-based PIs with and without UV light irradiation are displayed in Fig. 5 . The images without UV irradiation clearly show the colorless and transparency of the all the 6FDA-based PI films. In addition, only the 6FDA end-capped PI films exhibit obvious green fluorescence as discussed above.

To clarify the photophysical processes of 6FDA/DCHM/3HPA PIs, the fluorescence decay curves at $530 \mathrm{~nm}$ were measured with excitation at $340 \mathrm{~nm}$ (Fig. 6). The ESIPT fluorescence lifetime can be obtained by fitting analysis using exponential functions for decay curves (Table 1). It is clearly identified that all the decay curves are totally overlapped, and the obtained lifetimes $(\tau)$ are ranged in 4.9-5.0 ns without dependence on $r$. This result proves that $\tau$ is not influenced by the degree of polymerization of 6FDA-based PIs. In the previously reported ODPA-based PIs [1], the lifetime of ODPA/DCHM unit fluorescence decreased with increasing the $r$ ratio, which is the common case that the fluorescence lifetime of the donor moiety (ODPA/DCHM unit) decreases with increasing the acceptor moiety (3HPA), because of the enhanced efficiency of Förster resonance energy transfer (FRET) process from 
the main chain to the end-groups [21]. In contrast, for the 6FDA-based PIs, 6FDA/DCHM main chain exhibit almost no fluorescence as stated above. Thereby, no FRET process was generated between the main chain and end groups.

For further discussions of the fluorescence properties of 6FDA-based PIs, the quantum yields $(\Phi)$ of fluorescent emissions of the 6FDA-based and ODPA-based PIs [1] were compared as in Table 2. For the 6FDA-based PIs, the $\Phi$ value of a film prepared at $r=1.98$ is relatively low because of the insufficient amount of chromophore. In contrast, the other 6FDA-based PI films prepared at each $r$ value demonstrated significantly higher $\Phi$ values compared with the ODPA-based PI films, which is attributable to the suppression of FRET which causes non-radiative deactivation. In other words, the $-\mathrm{CF}_{3}$ groups restrict the formation of closely packed PI chains [22-28], which reduces interchain dipolar-dipolar interactions inducing the FRET. Moreover, the absence of FRET between the main chain and the end groups which due to the non-fluorescence 6FDA/DCHM unit also contributes to their high quantum yields. All these results proved that 6 FDA is a suitable and promising dianhydride for making colorless and highly fluorescent end-capped PIs.

\section{Conclusion}

A series of novel highly fluorescent polyimides (PIs) of 6FDA/DCHM/3HPA were synthesized, and their fluorescence properties have been investigated. The UV-vis absorption spectra and photo image of the films deposited on fused silica substrate clearly showed that all the 6FDA-based PI films display colorlessness with excellent transparency. In addition, 6FDA/DCHM/3HPA PIs exhibited prominent green fluorescence at $530 \mathrm{~nm}$ when excited at $340 \mathrm{~nm}$ despite no fluorescence properties in 6FDA/DCHM PI. This extraordinarily large Stokes shift $\left(10,544 \mathrm{~cm}^{-1}\right)$ is assignable to the fluorescence emitted from the excited keto form of 3HPA end groups via ESIPT process. All the 6FDA/DCHM/3HPA PIs demonstrated very similar fluorescence decay curves, which proves that these PIs have no dependence of fluorescence lifetime $\tau$ on the degree of polymerization. The quantum yields of the 6FDA-based PIs $(\Phi)$ were significantly higher than those of the ODPA-based PIs, which mainly due to the loosely packed PI chains and absence of FRET process. These results prove that 6FDA is a suitable and promising dianhydride for making colorless and highly fluorescent end-capped PIs.

\section{Acknowledgements}

This work was supported in part by Grants-in-Aid for Scientific Research, Japan Society for the Promotion of Science (17H03112), and N.L. thanks to the help of the China Scholarship Council (CSC).

\section{References}

1. J. Wakita, S. Inoue, N. Kawanishi, and S. Ando, Macromolecules, 43 (2010) 3594.

2. K. Kanosue, T. Shimosaka, J. Wakita, and S. Ando, Macromolecules, 48 (2015) 1777.

3. C. E. Sroog, J. Polym. Sci., Macromol. Rev., 11 (1976) 161.

4. K. Kanosue, R. Augulis, D. Peckus, R. Karpicz, T. Tamulevičius, S. Tamulevičius, V. Gulbinas, and S. Ando, Macromolecules, 49 (2016) 1848.

5. K. Kanosue and S. Ando, ACS Macro Lett., 5 (2016) 1301.

6. K. Kanosue, S. Hirata, M. Vacha, R. Augulis, V. Gulbinas, R. Ishige, and S. Ando, Mater. Chem. Front., 3 (2019) 39.

7. R. Orita, M. Franckevičius, A. Vyšniauskas, V. Gulbinas, H. Sugiyama, H. Uekusa, K. Kanosue, R. Ishige, and S. Ando, Phys. Chem. Chem. Phys., 20 (2018) 16033.

8. J. Wakita, H. Sekino, K. Sakai, Y. Urano, and S. Ando, J. Phys. Chem. B, 113 (2009) 15212.

9. A. Demeter, T. Bérces, L. Biczók, V. Wintgens, P. Valat, and J. Kossanyi, J. Phys. Chem., 100 (1996) 2001.

10. M. Hasegawa, M. Kochi, I. Mita, and R. Yokota, Eur. Polym. J., 25 (1989) 349.

11. J. Ishii, S. Horii, N. Sensui, M. Hasegawa, L. Vladimirov, M. Kochi, and R. Yokota, High Perform. Polym., 21 (2009) 282.

12. . Zhao and R. R. Lunt, Adv. Energy Mater, 3 (2013) 1143.

13. S. Ando, T. Matsuura, and S. Sasaki, Macromolecules, 25 (1992) 5858.

14. A. Weller, Z. Elektrochem. Ber. Bunsenges. Physik. Chem., 56 (1952) 662.

15. J. E. Kwon and S. Y. Park, Adv. Mater, 23 (2011) 3615.

16. L. He, L. Duan, J. Qiao, G. Dong, L. Wang, and Y. Qiu, Chem. Mater, 22 (2010) 3535.

17. T. Matsuura, S. Ando, S. Sasaki, and F. 
Yamamoto, Macromolecules, 27 (1994) 6665.

18. T. Matsumoto, High Perform. Polym., 11 (1999) 367.

19. Y. Oishi, K. Ogasawara, H. Hirahara, and K. Mori, J. Photopolym. Sci. Technol., 14 (2001) 37.

20. Y. Oishi, N. Kikuchi, K. Mori, S. Ando, and K. Maeda, J. Photopolym. Sci. Technol., 15 (2002) 213.

21. I. Yamazaki, N. Tami, and T. Yamazaki, $J$. Phys. Chem., 94 (1990) 516.

22. B. Liu, W. Hu, T. Matsumoto, Z. Jiang, and S. Ando, J. Polym. Sci. Part A: Polym. Chem., 43 (2005) 3018.
23. S. Ando, T. Sawada, and S. Sasaki, Polym. Adv. Technol., 12 (2001) 319.

24. Y. B. Zhuang and S. Ando, Polymer, 123 (2017) 39.

25. S. Miyata, S. Sato, K. Nagai, T. Nakagawa, and K. Kudo, J. Appl. Polym. Sci., 107 (2008) 3933.

26. Y. J. Cho and H. B. Park, Macromol. Rapid Commun., 32 (2011) 579.

27. D. Yorifuji and S. Ando, Macromolecules, 43 (2010) 7583.

28. P. K. Tapaswi, M. C. Choi, K. M. Jeong, S. Ando, and C. S. Ha, Macromolecules, 48 (2015) 3462. 